\title{
Space Charge Studies on Mid-voltage Cable by Thermally Stimulated Depolarization Currents in the Melting Temperature Range.
}

\author{
J.A Diego $^{1 *}$, J.C. Cañadas, J.Belana, J. Sellarès, J. Orrit, F. Frutos ${ }^{2}$ \\ ${ }^{1}$ Dept. de Física i E.N., ETSEIT, Universitat Politècnica de Catalunya. \\ C/Colom 11, 08222 Terrassa, Spain \\ ${ }^{2}$ Dept. de Física Aplicada I, ETSII, Universidad de Sevilla, \\ Avda. Reina de las Mercedes s/n, 41012 Sevilla, Spain \\ ${ }^{*}$ E-mail : jose.antonio.diego@upc.es
}

\begin{abstract}
In the present work, a XLPE mid-voltage cable from General Cable co. has been studied by Thermally stimulated depolarization currents. Systematic measurements have been carried out in order to compare the conductive processes in this cable with previous results. Depolarization current as a function of thermal annealing, thermal history, polarizing field and polarizing time and temperature has been obtained. The results show the presence of a broad and complex heteropolar process between 60 and $120^{\circ} \mathrm{C}$ as expected. Annealing of the sample at temperatures above $80^{\circ} \mathrm{C}$ develops an homopolar contribution associated to chemical components diffused from the cable semiconducting layers into the XLPE bulk. For annealing times of $60 \mathrm{~min}$ at $140^{\circ} \mathrm{C}$ and 2 days at $90^{\circ} \mathrm{C}$, the homoplar current intensity reaches a maximum, decreasing and recovering the heteropolar sign with further annealing. Experiments performed with different polarizing times and temperatures show as well the presence of an homopolar contribution, overlapped to the heteropolar behavior, that increases continuously with polarizing time. These results indicate that conductive processes within the XLPE are probably responsible of homopolar charge injection.
\end{abstract}

\section{INTRODUCTION}

The presence of space charge in a dielectric material not only affects the value of the electric field and its conductive properties, but also plays an important role in aging processes. From an applied point of view, in the case of mid-voltage cable, the study of space charge formation and relaxation processes is specially interesting as they condition cable lifetime. The working temperature of this cables, around $90^{\circ} \mathrm{C}$, is located in the melting temperature range, so the conduction processes observed are probably associated with free charge. Thermally stimulated depolarization currents (TSDC) has been widely used to study relaxation processes in polymers and has become a useful technique to determine the origin of these charges in cable [1].

Previous works in mid-voltage cable XLPE insulation by TSDC, have shown a rather complex behavior with three, highly overlapped, heteropolar current peaks located approximately at 80,95 and $105^{\circ} \mathrm{C}$ [2]. Together with these heteropolar peaks, an homopolar peak was detected at $98^{\circ} \mathrm{C}$ approximately, that is highly affected by the annealing condition of the material. This behavior, and specially the evolution of the homopolar contribution observed, was explained in terms of two separated processes responsible of the TSDC spectrum. One broad heteropolar contribution located between 60 and $120^{\circ} \mathrm{C}$, associated to the material melt, and a second process of homopolar character located at approximately $98^{\circ} \mathrm{C}$ that increases with annealing [3].

IR-FTIR measurements in cable showed that the evolution of the homopolar contribution was probably related to the diffusion of some chemical components from the isolation surface into the bulk [4]. During annealing at temperatures above $80^{\circ} \mathrm{C}$, an important fraction of the material is melt, and diffusion of these components can take place. The growth of the homopolar current was explained assuming that some of these components remain located in the surface of the crystalline spherulites and act as trapping centers during polarization. In any case, the complex behavior of the TSDC spectrum evidenced the need of more data and a systematic study in order to establish the origin of the peaks firmly.

Study of the TSDC spectrum in the melting temperature range with conventional polarization methods presents an important drawback. The whole thermal steps of the polarization process will affect the fusion and solidification processes of the material in different ways. To overcome 'this drawback, in this paper the study was also made by non isothermal windowing polarization (NIW). In this new polarization method, the sample is cooled down from the melt always at the same cooling rate, and the electric field is applied at the desired temperature interval. By this way we can analyze the TSDC response in different polarization conditions with identical thermal history in all cases.

In the present work we focus our attention in the study of a new mid-voltage cable from General Cable co. Several base components vary between this cable and the ones used in previous works. Systematic measurements have been carried out in order to compare the conductive processes in this cable with previous results and verify the validity of the stated model. TSDC response as a function of thermal annealing, thermal history, polarizing field and polarizing time and temperature has been obtained. 


\section{EXPERIMENTAL}

Cable samples were supplied by General Cable and consisted of a cylindrical insulating XLPE layer, with $5.5 \mathrm{~mm}$ and $10 \mathrm{~mm}$ of inner and outer radii respectively. The samples had two $1 \mathrm{~mm}$ thickness semiconductor layers (SC), in contact with the inner and the external surfaces of XLPE. The SC layer composition and insulating geometry vary between the present cable and the ones used in previous works.

Seven centimeters long samples were cut from cable for TSDC measurements. The semiconductor layers were used as electrodes. To avoid shortcircuits, the external layer was partially removed from the sample ends, leaving a $2 \mathrm{~cm}$ wide semiconducting strip centered in the sample. The experimental setup for these measurements is described in detail elsewhere [2]. $2^{\circ} \mathrm{C} / \mathrm{min}$ heating and cooling rate were used for all measurements.

Sample polarization was performed by two different methods. In the first method (windowing polarization method, WP), the potential $V_{p}$ is applied at a polarization temperature $t_{p}$ for a polarization time $t_{p}$ and, afterward, the sample is cooled down to a temperature $T_{0}$ switching off the electric field just before the sample cooling begins. This polarization method allows one to resolve complex relaxations into its elementary components, determining the structure of complex relaxation processes. To avoid the different thermal history effects in the fusion and solidification processes of the material, the non isothermal windowing polarization method (NIW) was also used. In this polarization method the sample is cooled down from the melt always at the same cooling rate, and the electric field is applied at the desired temperature interval.

\section{RESULTS AND DISCUSSION}

\section{TSDC results}

First, we explored the behavior of the TSCD spectrum by WP as we set larger polarization temperatures. The samples were cooled down from the melt $\left(140^{\circ} \mathrm{C}\right)$ to the polarization temperature and then polarized for $t_{p}=5 \mathrm{~min}$ and cooled down afterward without applied field.

The results (Fig. 1) demonstrate the presence of a broad spectrum composed by at least two overlapped peaks at 80 and $110^{\circ} \mathrm{C}$ (curves for $T_{p}<80^{\circ} \mathrm{C}$ in Fig. 1). In the case of curve obtained for $T_{p}=70^{\circ} \mathrm{C}$, a weak current peak at $90^{\circ} \mathrm{C}$ can be also observed. The intensity of the higher temperature peak increases as $T_{p}$ increases at the time the lower temperature peaks disappear for $T_{p}>100^{\circ} \mathrm{C}$.

As stated in previous works for similar cables [2], this spectrum must be associated to the melting process of crystalline spherulites within the material. This melting process takes place between 60 and $115^{\circ} \mathrm{C}$ with a

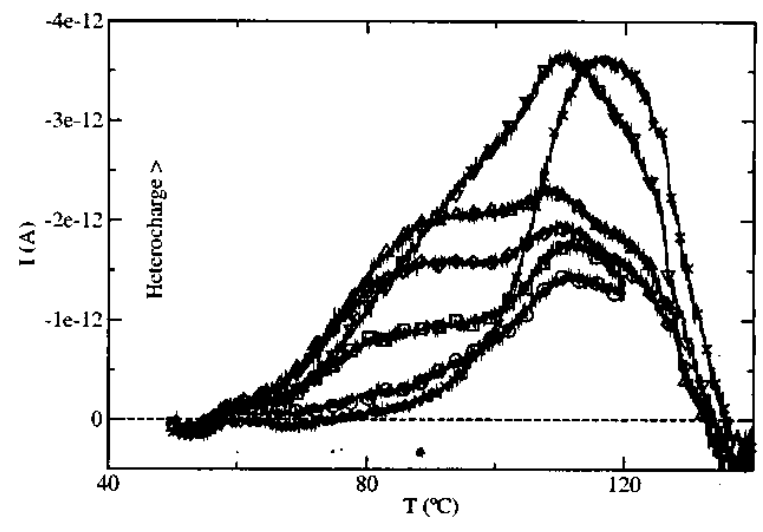

Fig. 1: WP-TSDC curves obtained cooling down from the melt to different $T_{p}: \circ 55^{\circ} \mathrm{C}, \square 65^{\circ} \mathrm{C}, \diamond 70^{\circ} \mathrm{C}, \triangle 75^{\circ} \mathrm{C}, \nabla 85^{\circ} \mathrm{C}$, $\times 105^{\circ} \mathrm{C}$. (processes: $T_{i}=140^{\circ} \mathrm{C}=>T_{p}\left(V_{p}=10 \mathrm{kV}, t_{p}=5 \mathrm{~min}\right.$ ) $\left.\Rightarrow T_{r}=50^{\circ} \mathrm{C}(5 \mathrm{~min})=>\mathrm{TSDC}\right)$.

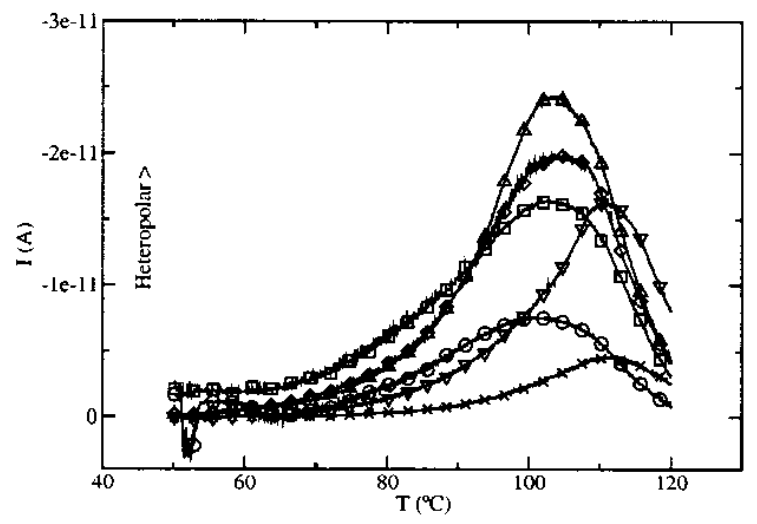

Fig. 2: WP-TSDC curves obtained heating up from room temperature to different $T_{p}: \circ 55^{\circ} \mathrm{C}, \square 60^{\circ} \mathrm{C}, \diamond 80^{\circ} \mathrm{C}, \triangle 90^{\circ} \mathrm{C}$, $\nabla 100^{\circ} \mathrm{C}, \times 110^{\circ} \mathrm{C}$. (processes: $T_{i}=35^{\circ} \mathrm{C}=>T_{p}\left(V_{p}=10 \mathrm{kV}\right.$, $\left.\left.t_{p}=5 \mathrm{~min}\right)=>T_{r}=50^{\circ} \mathrm{C}(5 \mathrm{~min})=>\mathrm{TSDC}\right)$.

maximum around $105^{\circ} \mathrm{C}$ as indicate differential scanning calorimetry results (DSC). As the material is progressively melt, polar charges induced during polarization in the crystal phase or in the interphases will release developing the observed discharge current.

Similar measurements were carried out by WP but in this case heating up the sample from room temperature to $T_{p}$, polarizing for $t_{p}=5 \mathrm{~min}$ and cooling down afterward without applied field. In this case (Fig. 2) a rather anomalous behavior is obtained, with much higher depolarization current intensity than in the previous case. A maximum in polarization is obtained as well for $T_{p}=90^{\circ} \mathrm{C}$. These remarkable results can be explained in terms of recrystallization processes that take place when the material is partially melted and annealed at temperatures within the melting range. More detailed study of this behavior will be the subject of future work. 


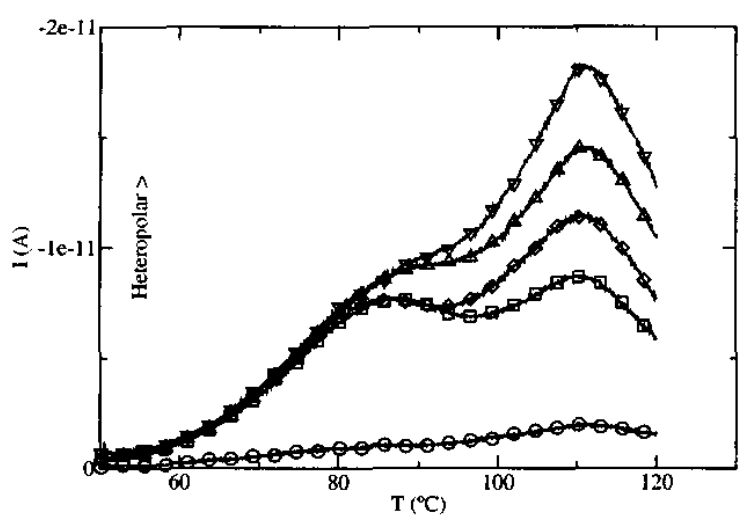

Fig. 3: NIW-TSDC curves obtained with samples starting polarization at different $T_{p}: \circ 60^{\circ} \mathrm{C}, \square 80^{\circ} \mathrm{C}, \diamond 90^{\circ} \mathrm{C}, \triangle 100^{\circ} \mathrm{C}$, $\nabla 110^{\circ} \mathrm{C}$. (processes: $T_{0}=35^{\circ} \mathrm{C}=>140^{\circ} \mathrm{C}=>T_{p}\left(V_{p}=10 \mathrm{kV}\right.$ ) $=>T_{r}=50^{\circ} \mathrm{C}$ (cooling with applied field $)=>$ TSDC).

To overcome this drawback related to the thermal history of each sample, a study of the spectrum was also made by the NIW polarization method (Fig. 3). In this case the samples were cooled down from $140^{\circ} \mathrm{C}$ to $50^{\circ} \mathrm{C}$ at $2^{\circ} \mathrm{C} / \mathrm{min}$ in all cases. The electric field was applied during cooling at $T_{p}\left(60,80,90\right.$, and $110^{\circ} \mathrm{C}$ respectively) and maintained until the sample reaches $50^{\circ} \mathrm{C}$. By this way all cables will have identical thermal history previous to measurement.

Obtained results demonstrate in this case as well the presence of a broad spectrum that extends along all the melting temperature range of XLPE. Only two overlapped peaks were detected in this case at 80 and $110^{\circ} \mathrm{C}$, indicating that the weak current peak detected at $90^{\circ} \mathrm{C}\left(T_{p}=70^{\circ} \mathrm{C}\right.$ in Fig. 1) is probably originated by the thermal process carried out during polarization.

\section{Evolution of TSDC discharges with thermal annealing}

We have annealed samples at 90 and $140^{\circ} \mathrm{C}$ for annealing times between 0 and 600 hours. These samples were then polarized by the NIW method, between $T_{p}=110^{\circ} \mathrm{C}$ and $50^{\circ} \mathrm{C}$, and the TSDC discharges measured afterward.

Fig. 4 show the TSDC discharges obtained with the sample annealed at $90^{\circ} \mathrm{C}$. As an overall trend we can observe, in the curve corresponding to the low annealed sample, the presence of two heteropolar peaks at 85 and $115^{\circ} \mathrm{C}$. With further annealing the $85^{\circ} \mathrm{C}$ peak shifts up to $95^{\circ} \mathrm{C}$. An homopolar peak appears after one hour annealing at this temperature, and its height decreases again with further annealing, disappearing after 1 day annealing. The same evolution but at a slower rate is observed in samples annealed at $90^{\circ} \mathrm{C}$ up to 192 hours (not plotted). The homopolar peak appears after two days annealing at this temperature, and its height decreases again with further annealing. In this case, however, the homopolar current

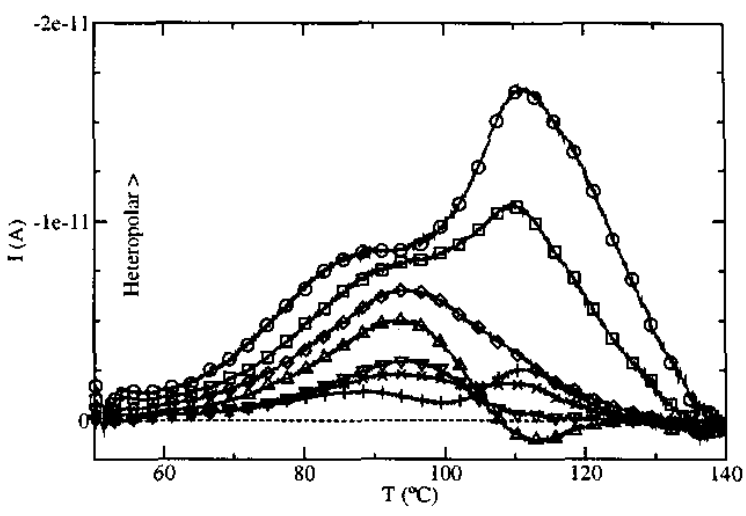

Fig. 4: NIW-TSDC curves obtained with samples aged at $140^{\circ} \mathrm{C}$ for different times: $\diamond 5 \mathrm{~min}, \square 15 \mathrm{~min}, \diamond 30 \mathrm{~min}, \Delta 1$ hour, $\nabla 6$ hours, $\times 1$ day, +2 days. (processes: $T_{0}=35^{\circ} \mathrm{C} \Rightarrow$ $140^{\circ} \mathrm{C} \Rightarrow>T_{p}=110^{\circ} \mathrm{C}\left(V_{p}=10 \mathrm{kV}\right)=>T_{r}=50^{\circ} \mathrm{C}$ (cooling with applied field) $=>$ TSDC).

does not fully disappears after 8 days annealing. The observed behavior is in good agreement with previous results obtained in other cables [4].

The generation of an homopolar current with annealing of the cable samples, and the subsequent described evolution, can be conveniently explained in the frame work of the model stated in previous works: IR-FTIR measurements showed that the evolution of the homopolar contribution is probably related to the diffusion of some chemical components from the isolation surface into the bulk [4]. This chemical components are mainly present in the surface of the XLPE isolation after manufacture. During annealing at temperatures above $80^{\circ} \mathrm{C}$, an important fraction of the material is melt, and diffusion of these components into the bulk can take place.

The appearance of the homopolar contribution was related to the creation of trapping centers in the bulk of the XLPE. The chemical components diffused from the surface into the isolation remain probably located in the surface of the spherulites and act as trapping centers during polarization. When the trapping center density is high (in samples annealed for long times) the polarizing field limits the homopolar contribution, that tends to saturation, and a maximum in the homoplar current is observed. Besides the described process that lead to the homopolar contribution, free charge is generated continuously in the material. This generation of free carriers predominates for long annealing times promoting the observed heteropolar current increase.

\section{Evolution of TSDC discharges with polarization time}

To analyze the effect of the electric field in the TSDC spectrum, cable samples were polaryzed according to the following process; the samples were cooled down from $140^{\circ} \mathrm{C}$ to the polarization temperature $T_{p}$ and polaryzed 


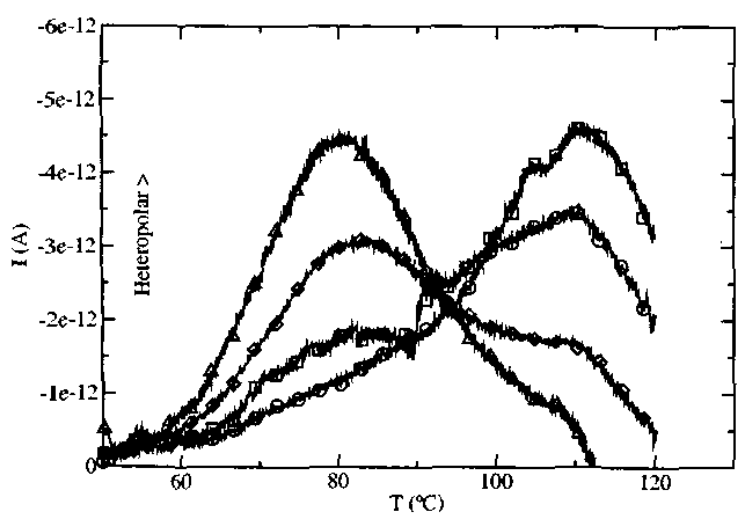

Fig. 5: TSDC curves obtained polarizing at $T_{p}=55^{\circ} \mathrm{C}$ for different polarization times $t_{p}: \circ 5 \mathrm{~min}, \square 30 \mathrm{~min}, \diamond 60 \mathrm{~min}$, $\triangle 120 \mathrm{~min}$. (processes: $T_{0}=140^{\circ} \mathrm{C}=>T_{p}=55^{\circ} \mathrm{C}\left(V_{p}=10 \mathrm{kV}, t_{p}\right)$ $=>T_{r}=50^{\circ} \mathrm{C}$ (cooling with applied field) $=>$ TSDC).

afterward for different times $t_{p}$. The sample was then cooled down to $50^{\circ} \mathrm{C}$ without switching off the electric field, and the the TSDC curves were measured.

We can observe in figures 5 and 6 the curves obtained for $T_{p}=55^{\circ} \mathrm{C}$ and $T_{p}=90^{\circ} \mathrm{C}$. In both cases the two overlapped relaxation processes at 80 and $110^{\circ} \mathrm{C}$ are detected, however they present rather different evolution with polarization time $t_{p}$. The $80^{\circ} \mathrm{C}$ peak show a continuous increase as higher polarization times are applied. This increase, coherent with the polarization process carried out, contrast with the observed evolution in the $110^{\circ} \mathrm{C}$ peak. In this case the intensity current grows initially, but with further increase in $t_{p}$, it decreases again obtaining a maximum in discharge current for $t_{p}=30 \mathrm{~min}$ and $t_{p}=60 \mathrm{~min}$ when $T_{p}=55^{\circ} \mathrm{C}$ and $T_{p}=90^{\circ} \mathrm{C}$ respectively. This evolution is better seen for $T_{p}=55^{\circ} \mathrm{C}$, as for $T_{p}=90^{\circ} \mathrm{C}$ the intensity of the heteropolar $110^{\circ} \mathrm{C}$ peak is high and interfere its observation.

This behavior indicates that the injection of charge in the material takes place in a continuous way during polarization, probably associated to a conducting process. Even more remarkable is the fact that this behavior is observed in not annealed samples indicating that some diffusion of chemical components from the surface towards the bulk of XLPE had already occurred during the manufacture process.

\section{CONCLUSIONS}

A rather complex TSDC spectrum is obtained in XLPE mid voltage cable isolation between 50 and $120^{\circ} \mathrm{C}$, which shows the development of an homopolar current contribution located around $110^{\circ} \mathrm{C}$. The evolution of this homopolar current is probably related to the diffusion of some chemical components from the isolation surface into the bulk during annealing at temperatures above $80^{\circ} \mathrm{C}$. At

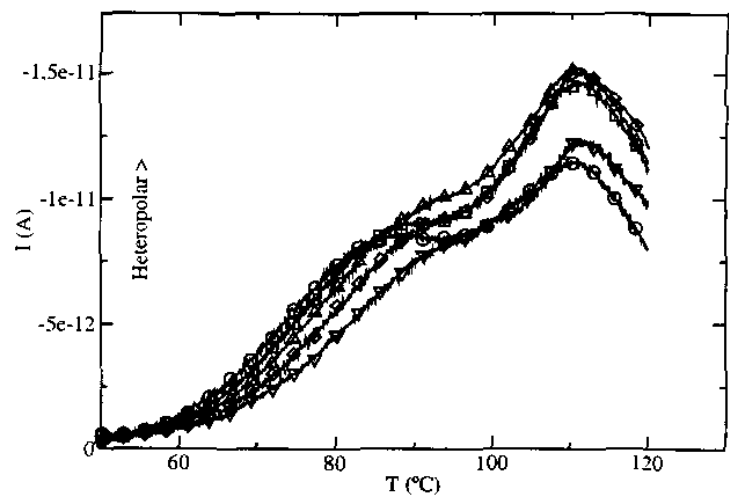

Fig. 6: TSDC curves for $T_{p}=90^{\circ} \mathrm{C}$ and different polarization times $t_{p}: \circ 5 \mathrm{~min}, \square 30 \mathrm{~min}, \diamond 60 \mathrm{~min}, \triangle 90 \mathrm{~min}, \nabla 120$ min. (processes: $T_{0}=140^{\circ} \mathrm{C} \Rightarrow>T_{p}=90^{\circ} \mathrm{C}\left(V_{p}=10 \mathrm{kV}, t_{p}\right)=>$ $T_{r}=50^{\circ} \mathrm{C}$ (cooling with applied field) $=>$ TSDC).

this temperatures an important fraction of the material is melt, and diffusion of these components into the bulk can take place. The initial increase of the homopolar current is explained in terms of a continuous increase of tapping centers as the components diffused into the insulator bulk. When the trap density is large enough the applied field determines the charge injection process and the largest homopolar peak is observed. Results obtained with different polarization times indicate that injected charge is not corona originated, but penetrates in the material probably due to a conduction process. The increase of the hetoropolar contribution with further annealing indicates that free carriers are produced continuously in the material. These results are in good agreement with the modelization of the conducting processes propounded in previous works.

\section{REFERENCES}

[1] R. Gerhard-Multhaupt, Electrets, third edition, Vol 2, (chapter 9). Morgan Hill, California, USA: Laplacian Press, 1999.

[2] I.Tamayo, J.Belana, J.C.Cañadas, J.A.Diego, M.Mudarra, J.Sellarés. J.Pol.Sci. Part B, Pol Phys Ed. vol 41,pp 1412-1421, 1993.

[3] I. Tamayo, M. Mudarra, J.A. Diego, J.C. Cañadas, J. Sellarès, J. Belana, in APTDAM'2001 Proceedings, (Wroclaw, Poland), pp. 84-87, 2001.

[4] I. Tamayo, J. Belana, J.A. Diego, J.C. Cañadas, M. Mudarra and J. Sellarès, Space charge studies in XLPE mid-voltage cable isolation by TSDC, IR-FTIR and $S E M$, submitted to Journal of Polymers Science Part B: Polymer Physics.

Acknowledgments: Present work is financed by ID project Ref MAT 2001-2338-C02-01 (MCT Spain). The authors wish to thank General Cable for their contribution to the work (cable samples and useful suggestions). 